\title{
Phase Transitions, Geometrothermodynamics, and Critical Exponents of Black Holes with Conformal Anomaly
}

\author{
Jie-Xiong Mo ${ }^{1,2}$ and Wen-Biao Liu ${ }^{1}$ \\ ${ }^{1}$ Department of Physics, Institute of Theoretical Physics, Beijing Normal University, Beijing 100875, China \\ ${ }^{2}$ Institute of Theoretical Physics, Zhanjiang Normal University, Zhanjiang 524048, China \\ Correspondence should be addressed to Wen-Biao Liu; wbliu@bnu.edu.cn
}

Received 9 December 2013; Accepted 3 February 2014; Published 13 March 2014

Academic Editor: George Siopsis

Copyright (c) 2014 J.-X. Mo and W.-B. Liu. This is an open access article distributed under the Creative Commons Attribution License, which permits unrestricted use, distribution, and reproduction in any medium, provided the original work is properly cited. The publication of this article was funded by $\mathrm{SCOAP}^{3}$.

\begin{abstract}
We investigate the phase transitions of black holes with conformal anomaly in canonical ensemble. Some interesting and novel phase transition phenomena have been discovered. It is shown that there are striking differences in both Hawking temperature and phase structure between black holes with conformal anomaly and those without it. Moreover, we probe in detail the dependence of phase transitions on the choice of parameters. The results show that black holes with conformal anomaly have much richer phase structure than those without it. There would be two, only one, or no phase transition points depending on the parameters. The corresponding parameter regions are derived both numerically and graphically. Geometrothermodynamics are built up to examine the phase structure we have discovered. It is shown that Legendre invariant thermodynamic scalar curvature diverges exactly where the specific heat diverges. Furthermore, critical behaviors are investigated by calculating the relevant critical exponents. And we prove that these critical exponents satisfy the thermodynamic scaling laws.
\end{abstract}

\section{Introduction}

Black hole thermodynamics has long been one of the exciting and challenging research fields ever since the pioneer research made by Bekenstein and Hawking $[1,2]$. A variety of thermodynamic quantities of black holes have been studied. In 1983, Hawking and Page [3] discovered that pure thermal radiation in AdS space becomes unstable above certain temperature and collapses to form black holes. This is the well-known Hawking-Page phase transition which describes the phase transition between the Schwarzschild AdS black hole and the thermal AdS space. This phenomenon can be interpreted in the AdS/CFT correspondence [4] as the confinement/deconfinement phase transition of gauge field [5]. Since then, phase transitions of black holes have been investigated from different perspectives. For recent papers, see [6-26].

One of the elegant approaches is the thermodynamic geometry method, which was first introduced by Weinhold [27] and Ruppeiner [28]. Weinhold proposed metric structure in the energy representation as $g_{i, j}^{W}=$ $\partial_{i} \partial_{j} M\left(U, N^{a}\right)$ while Ruppeiner defined metric structure as $g_{i, j}^{R}=-\partial_{i} \partial_{j} S\left(U, N^{a}\right)$. These metric structures are, respectively, the Hessian matrix of the internal energy $U$ and the entropy $S$ with respect to the extensive thermodynamic variables $N^{a}$. And Weinhold's metrics were found to be conformally connected to Ruppeiner's metrics through the map $d S_{R}^{2}=d S_{W}^{2} / T$ [29]. Ruppeiner's metric has been applied to investigate various thermodynamics systems for its profound physical meaning. For more details, see the nice review in [30]. Recently, Quevedo [31] presented a new formalism called geometrothermodynamics, which allows us to derive Legendre invariant metrics in the space of equilibrium states. Geometrothermodynamics presents a unified geometry where the metric structure describes various types of black hole thermodynamics [32-41].

Apart from the thermodynamic geometry, critical behavior also plays a crucial role in the study of black hole phase transitions. The critical points of phase transitions are characterized by the discontinuity of thermodynamic quantities. So, it is important to investigate the behavior 
in the neighborhood of the critical point, especially the divergences of various thermodynamic quantities. In classical thermodynamics, this goal is achieved by taking into account a set of critical exponents, from which we can gain qualitative insights into the critical behavior. These critical exponents are found to be universal to a large extent (only depending on the dimensionality, symmetry, etc.) and satisfy scaling laws, which can be attributed to scaling hypothesis. Critical behavior of black holes accompanied with their critical exponents has been investigated not only in asymptotically flat space time [42-48] but also in the de Sitter and anti-de Sitter space [49-55].

In this paper, we would like to focus our attention on the critical behavior and geometrothermodynamics of static and spherically symmetric black holes with conformal anomaly. As we know, conformal anomaly, an important concept with a long history, has various applications in quantum field theory in curved spaces, string theory, black hole physics, and cosmology. So it is worth probing its influences in phase transitions of black holes. Recently, Cai et al. [56] found a class of static and spherically symmetric black holes with conformal anomaly, whose thermodynamic quantities were also investigated in the same paper. It was found that there exists a logarithmic correction to the well-known BekensteinHawking area entropy. This discovery is quite important in the sense that with this term one is able to compare black hole entropy up to the subleading order, in the gravity side and in the microscopic statistical interpretation side [56]. Based on the metrics in that paper, phase transitions of a spherically symmetric Schwarzschild black hole have been investigated by taking into account the back reaction through the conformal anomaly of matter fields recently [57]. It has been shown that there exists an additional phase transition to the conventional Hawking-Page phase transition. The entropy of these black holes has also been investigated by using quantum tunneling approach [58]. Moreover, Ehrenfest equation has been applied to investigate this class of black holes [59] and it has been found that the phase transition is a second-order one. Despite these achievements, there are still many issues left to be explored. Reference [57] mainly focuses on the uncharged case. So, it is natural to ask what would happen to the charged black holes. In [59], the authors concentrated their efforts on the Ehrenfest equation in the grand canonical ensemble. So it is worthwhile to study the phase transition in canonical ensemble. The dependence of the phase structure on the parameter deserves to be further investigated. One may also wonder whether the thermodynamic geometry and scaling laws still work to reveal the phase structure and critical behavior when conformal anomaly is taken into consideration. Motivated by these, we would like to investigate the phase transition, geometrothermodynamics, and critical exponents in canonical ensemble.

The organization of our paper is as follows. In Section 2, the thermodynamics of black holes with conformal anomaly will be briefly reviewed. In Section 3, phase transitions will be investigated in detail in canonical ensemble, and some interesting and novel phase transition phenomena will be disclosed. In Section 4, geometrothermodynamics will be established to examine the phase structure we find in
Section 3. In Section 5, critical exponents will be calculated and the scaling laws will be examined. In the end, conclusions will be drawn in Section 6 .

\section{A Brief Review of Thermodynamics}

The static and spherically symmetric black hole solution with conformal anomaly has been proposed as [56]

$$
d s^{2}=f(r) d t^{2}-\frac{d r^{2}}{f(r)}-r^{2}\left(d \theta^{2}+\sin ^{2} \theta d \varphi^{2}\right),
$$

where

$$
f(r)=1-\frac{r^{2}}{4 \widetilde{\alpha}}\left(1-\sqrt{1-\frac{16 \tilde{\alpha} M}{r^{3}}+\frac{8 \widetilde{\alpha} Q^{2}}{r^{4}}}\right) .
$$

The Newton constant $G$ has been set to one. Both $M$ and $Q$ are integration constants. And the coefficient $\widetilde{\alpha}$ is positive. The physical meanings of $M$ and $Q$ were discussed in [56]. $M$ is nothing but the mass of the solution while $Q$ should be interpreted as $U(1)$ charge of some conformal field theory.

When $M=Q=0$, the metric above reduces to

$$
d s^{2}=d t^{2}-d r^{2}-r^{2}\left(d \theta^{2}+\sin ^{2} \theta d \varphi^{2}\right),
$$

implying that the vacuum limit is the Minkowski space-time. In the large $r$ limit, (2) becomes

$$
f(r) \approx 1-\frac{2 M}{r}+\frac{Q^{2}}{r^{2}}+O\left(r^{-2}\right),
$$

which behaves like the Reissner-Nordström solution.

When $\widetilde{\alpha} \rightarrow 0$, (2) reduces into

$$
f(r)=1-\frac{2 M}{r}+\frac{Q^{2}}{r^{2}} .
$$

Equations (1) and (5) consist of the metric of ReissnerNordström black hole.

Solving the equation $f(r)=0$, we can get the radius of black hole horizon $r_{+}$, with which the mass of the black hole can be expressed as

$$
M=\frac{r_{+}}{2}+\frac{Q^{2}}{2 r_{+}}-\frac{\widetilde{\alpha}}{r_{+}} .
$$

The Hawking temperature can be derived as

$$
T=\frac{f^{\prime}\left(r_{+}\right)}{4 \pi}=\frac{r_{+}^{2}+2 \widetilde{\alpha}-Q^{2}}{4 \pi r_{+}\left(r_{+}^{2}-4 \widetilde{\alpha}\right)} .
$$

The potential difference between the horizon and the infinity can be written as

$$
\Phi=\frac{Q}{r_{+}} .
$$

The entropy was reviewed in [59] as

$$
S=\pi r_{+}^{2}-4 \pi \tilde{\alpha} \ln r_{+}^{2} .
$$




\section{Novel Phase Transition Phenomena}

In this section, we would like to investigate the phase transition of black holes with conformal anomaly in canonical ensemble where the charge of the black hole is fixed.

The corresponding specific heat can be calculated as

$$
C_{Q}=T\left(\frac{\partial S}{\partial T}\right)_{Q}=\frac{2 \pi\left(r_{+}^{2}-4 \widetilde{\alpha}\right)^{2}\left(Q^{2}-2 \tilde{\alpha}-r_{+}^{2}\right)}{r_{+}^{4}-8 \widetilde{\alpha}^{2}+10 r_{+}^{2} \tilde{\alpha}+Q^{2}\left(4 \widetilde{\alpha}-3 r_{+}^{2}\right)}
$$

Apparently, $C_{Q}$ may diverge when

$$
r_{+}^{4}-8 \widetilde{\alpha}^{2}+10 r_{+}^{2} \widetilde{\alpha}+Q^{2}\left(4 \widetilde{\alpha}-3 r_{+}^{2}\right)=0
$$

which suggests a possible phase transition. However, the phase transition point characterized by (11) is not apparent. To gain an intuitive understanding, we plot Figure 1(a) using (10). To check whether the phase transition point locates in the physical region, we also plot the Hawking temperature using (7) in Figure 1(b). It is shown that the phase transition point locates in the positive temperature region. From Figures $1(\mathrm{a})$ and $1(\mathrm{~b})$, we find that there have been striking differences between the case $\widetilde{\alpha} \neq 0$ and the case $\widetilde{\alpha}=0$. In the case $Q=1, \widetilde{\alpha}=0.1$, there are two phase transition points while there is only one in the case $\widetilde{\alpha}=0$. The temperature in the case $\widetilde{\alpha}=0$ increases monotonically while there exists local minimum temperature in the case $Q=1, \widetilde{\alpha}=0.1$. Figure 1(a) can be divided into four phases. The first one is thermodynamically stable $\left(C_{Q}>0\right)$ with small radius. The second one is unstable $\left(C_{Q}<0\right)$ with medium radius. The third one is thermodynamically stable $\left(C_{Q}>0\right)$ with medium radius while the fourth one is thermodynamically unstable $\left(C_{Q}<0\right)$ with large radius. So the phase transition takes place not only from an unstable large black hole to a locally stable medium black hole but also from an unstable medium black hole to a locally stable small black hole. From Figure 1(b), we notice that the Hawking temperature has a local minimum value. And the corresponding location can be derived through

$$
\frac{\partial T}{\partial r_{+}}=-\frac{r_{+}^{4}-8 \widetilde{\alpha}^{2}+10 r_{+}^{2} \widetilde{\alpha}+Q^{2}\left(4 \widetilde{\alpha}-3 r_{+}^{2}\right)}{4 \pi\left(r_{+}^{3}-4 r_{+} \widetilde{\alpha}\right)^{2}}=0 .
$$

It is quite interesting to note that the numerator of (12) is the same as (11), which implies that the location which corresponds to the minimum Hawking temperature also witnesses the existence of phase transition.

To probe the dependence of phase transition location on the choice of parameter, we solve (11) and obtain the location of phase transition point as

$$
r_{c}=\sqrt{\frac{3 Q^{2}-10 \widetilde{\alpha} \pm \sqrt{132 \widetilde{\alpha}^{2}-76 \widetilde{\alpha} Q^{2}+9 Q^{4}}}{2}} .
$$

With (13) at hand, we plot Figures 2(a) and 2(b) which show the influence of parameters $Q$ and $\widetilde{\alpha}$, respectively. It can be observed from Figures 2(a) and 2(b) that black holes with conformal anomaly have much richer phase structure than that without conformal anomaly. When $\tilde{\alpha}=0$, the location of the phase transition $r_{c}$ is proportional to the charge $Q$. However, the cases of black holes with conformal anomaly are quite complicated. For $\widetilde{\alpha}=0.1$, the curve can be divided into three regions. Through numerical calculation, we find that black holes have only one phase transition point when $Q \subset(0,0.4472)$. When $0.4472<Q<0.7746$, there would be no phase transition at all. When $Q>0.7746$, there exist two phase transition points, just as what we show in Figure 1(a). And the distance between these two phase transition points becomes larger with the increasing of $Q$. Figure 2(b) shows the case that the charge $Q$ has been fixed at one. We notice that there would be two phase transition points when $0<\widetilde{\alpha}<$ $1 / 6$, which is consistent with Figure 1(a). And the distance between these two phase transition points becomes narrower with the increasing of $\widetilde{\alpha}$. When $\widetilde{\alpha} \subset(1 / 6,1 / 2)$, there would be no phase transition. When $\tilde{\alpha}>1 / 2$, there would be only one phase transition point. To gain a three-dimensional understanding, we also include a three-dimensional figure of $C_{Q}$ in Figures 3(a) and 3(b).

Apart from the specific heat, we would also like to investigate the behavior of the inverse of the isothermal compressibility, which is defined as

$$
\kappa_{T}^{-1}=Q\left(\frac{\partial \Phi}{\partial Q}\right)_{T} .
$$

Utilizing the thermodynamic identity relation

$$
\left(\frac{\partial \Phi}{\partial T}\right)_{Q}\left(\frac{\partial T}{\partial Q}\right)_{\Phi}\left(\frac{\partial Q}{\partial \Phi}\right)_{T}=-1,
$$

we obtain

$$
\left(\frac{\partial \Phi}{\partial Q}\right)_{T}=-\left(\frac{\partial \Phi}{\partial T}\right)_{Q}\left(\frac{\partial T}{\partial Q}\right)_{\Phi}
$$

where the second term on the right hand side can be calculated through

$$
\left(\frac{\partial T}{\partial Q}\right)_{\Phi}=\left(\frac{\partial T}{\partial r_{+}}\right)_{Q}\left(\frac{\partial r_{+}}{\partial Q}\right)_{\Phi}+\left(\frac{\partial T}{\partial Q}\right)_{r_{+}} .
$$

Utilizing (7), (8), (14), (16), and (17), we obtain the explicit form of $\kappa_{T}^{-1}$ as

$$
\kappa_{T}^{-1}=\frac{Q r_{+}^{4}-Q^{3} r_{+}^{2}-4 Q^{3} \tilde{\alpha}+10 Q r_{+}^{2} \tilde{\alpha}-8 Q \widetilde{\alpha}^{2}}{r_{+}\left[r_{+}^{4}-8 \widetilde{\alpha}^{2}+10 r_{+}^{2} \tilde{\alpha}+Q^{2}\left(4 \widetilde{\alpha}-3 r_{+}^{2}\right)\right]} .
$$

We show the behavior of $\kappa_{T}^{-1}$ in Figure 4. Comparing Figure 4 with Figure 1(a), we find that the inverse of the isothermal compressibility $\kappa_{T}^{-1}$ also diverges at the critical point.

\section{Geometrothermodynamics}

According to geometrothermodynamics [31], the $(2 n+1)$ dimensional thermodynamic phase space $\mathscr{T}$ can be coordinated by the set of independent quantities $\left\{\phi, E^{a}, I^{a}\right\}$, where 


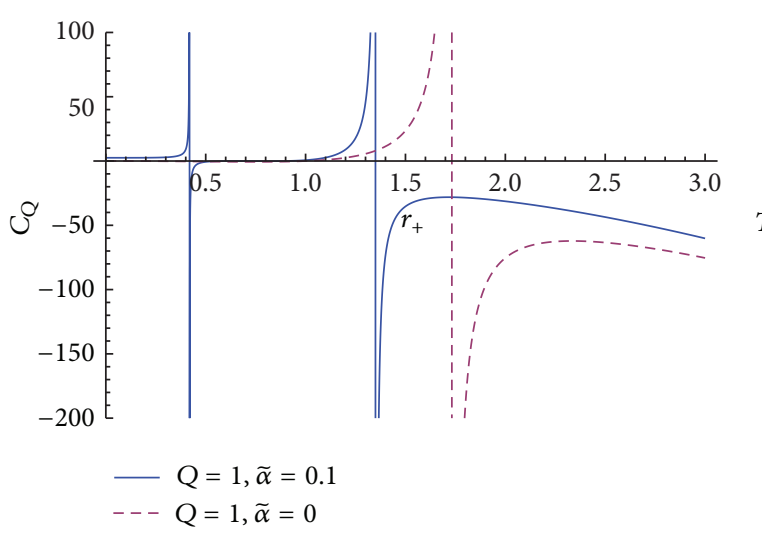

(a)

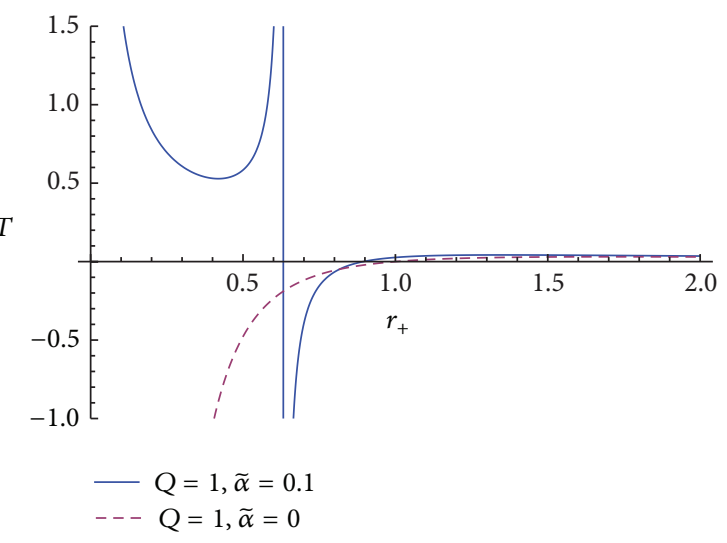

(b)

Figure 1: (a) $C_{Q}$ versus $r_{+}$for $Q=1, \widetilde{\alpha}=0.1$. (b) $T$ versus $r_{+}$for $Q=1, \widetilde{\alpha}=0.1$.

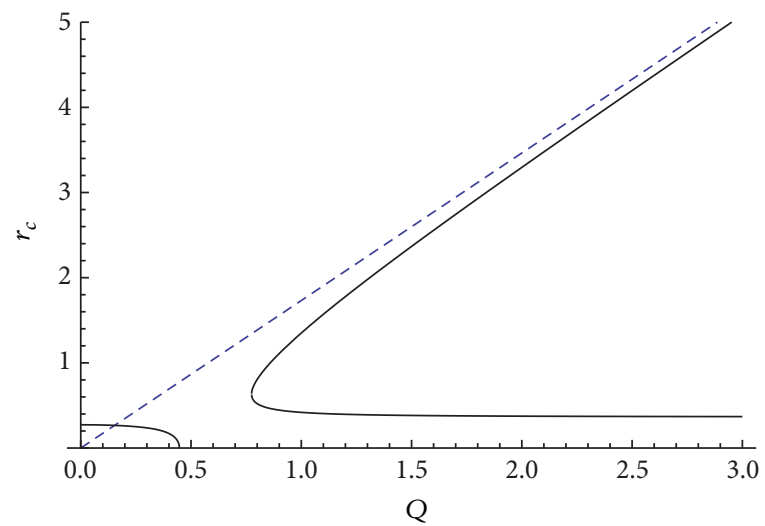

$--\tilde{\alpha}=0$

$-\widetilde{\alpha}=0.1$

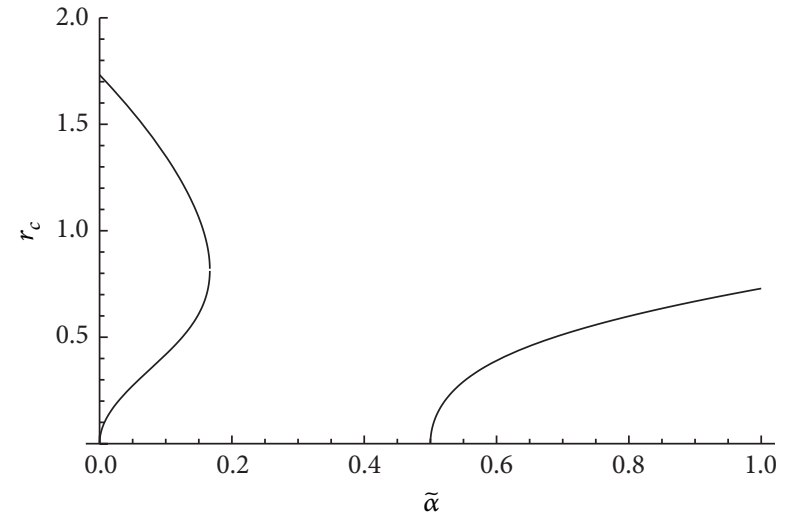

(b)

(a)

Figure 2: (a) $r_{c}$ versus $Q$ for $\widetilde{\alpha}=0.1$ and $\widetilde{\alpha}=0$. (b) $r_{c}$ versus $\widetilde{\alpha}$ for $Q=1$.

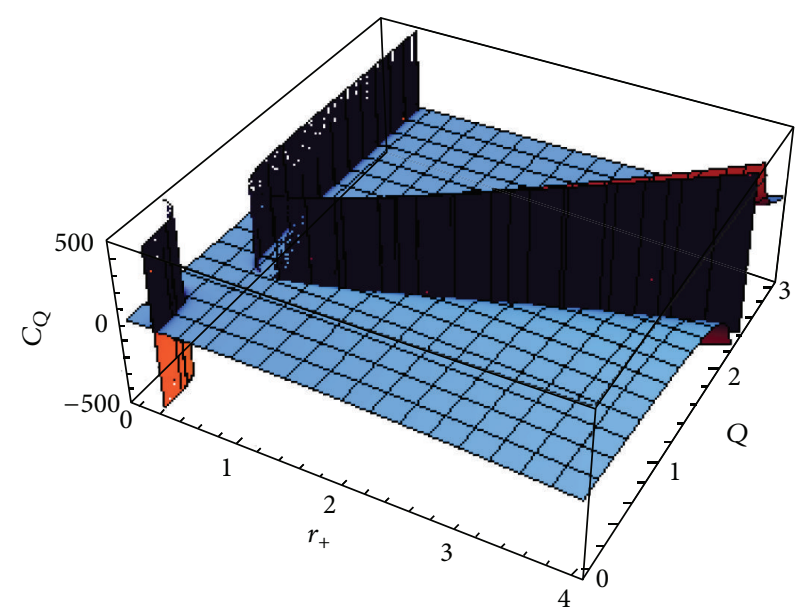

(a)

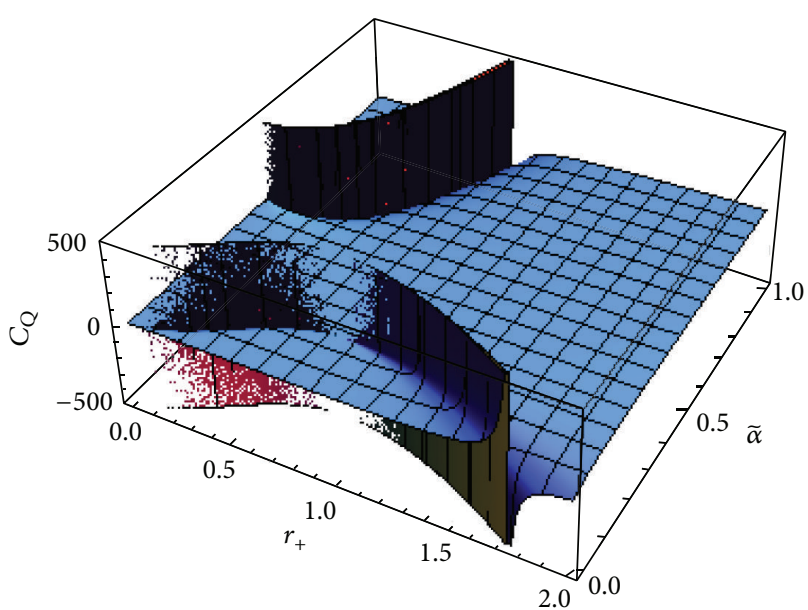

(b)

Figure 3: (a) $C_{Q}$ versus $Q$ and $r_{+}$for $\widetilde{\alpha}=0.1$. (b) $C_{Q}$ versus $\widetilde{\alpha}$ and $r_{+}$for $Q=1$. 


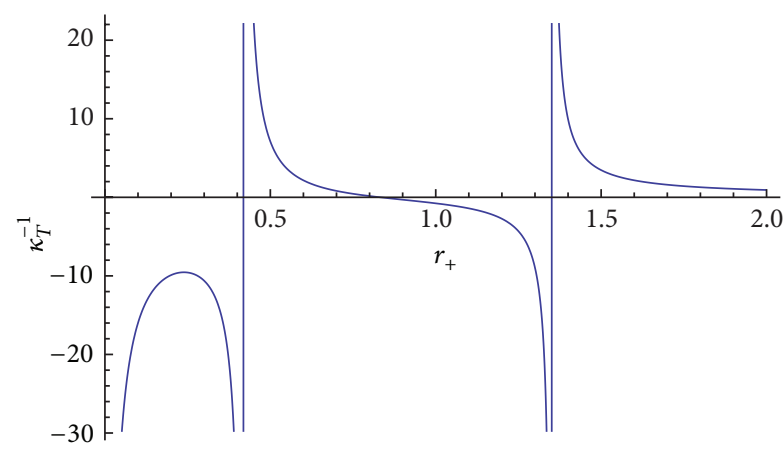

Figure 4: The inverse of the isothermal compressibility $\kappa_{T}^{-1}$ versus $r_{+}$for $Q=1, \widetilde{\alpha}=0.1$.

$\phi$ corresponds to the thermodynamic potential and $E^{a}, I^{a}$ are the extensive and intensive thermodynamic variables, respectively. The fundamental Gibbs 1-form defined on $\mathscr{T}$ can then be written as $\Theta=d \phi-\delta_{a b} I^{a} d E^{b}$, where $\delta_{a b}=$ $\operatorname{diag}(1, \ldots, 1)$. Considering a nondegenerate Riemannian metric $G$, a contact Riemannian manifold can be defined from the set $(\mathscr{T}, \Theta, G)$ if the condition $\Theta \wedge(\Theta)^{n} \neq 0$ is satisfied. Utilizing a smooth map $\varphi: \varepsilon \rightarrow \mathscr{T}$, that is, $\varphi:\left(E^{a}\right) \mapsto\left(\phi, E^{a}, I^{a}\right)$, a submanifold $\varepsilon$ called the space of thermodynamic equilibrium states can be induced. Furthermore, a thermodynamic metric $g$ can be induced in the equilibrium manifold $\varepsilon$ by the smooth map $\varphi$.

As proposed by Quevedo et al., the nondegenerate metric $G$ and the thermodynamic metric $g$ can be written as follows [38]:

$$
\begin{gathered}
G=\left(d \phi-\delta_{a b} I^{a} d E^{b}\right)^{2}+\left(\delta_{a b} E^{a} I^{b}\right)\left(\eta_{c d} d E^{c} d I^{d}\right), \\
g=\varphi^{*}(G)=\left(E^{c} \frac{\partial \phi}{\partial E^{c}}\right)\left(\eta_{a b} \delta^{b c} \frac{\partial^{2} \phi}{\partial E^{c} \partial E^{d}} d E^{a} d E^{d}\right),
\end{gathered}
$$

where $\eta_{a b}=\operatorname{diag}(-1, \ldots, 1)$.

To construct geometrothermodynamics of black holes with conformal anomaly in canonical ensemble, we choose $M$ to be the thermodynamic potential and $S, Q$ to be the extensive variables. Then the corresponding thermodynamic phase space is a 5 -dimensional one coordinated by the set of independent coordinates $\{M, S, Q, T, \Phi\}$. The fundamental Gibbs 1-form defined on $\mathscr{T}$ can then be written as

$$
\Theta=d M-T d S-\Phi d Q .
$$

The nondegenerate metric $G$ from (19) can be changed into

$$
G=(d M-T d S-\Phi d Q)^{2}+(T S+\Phi Q)(-d S d T+d Q d \Phi) .
$$

Introducing the map

$$
\varphi:\{S, Q\} \longmapsto\left\{M(S, Q), S, Q, \frac{\partial M}{\partial S}, \frac{\partial M}{\partial Q}\right\},
$$

the space of thermodynamic equilibrium states can be induced. According to (19), the thermodynamic metric $g$ can be written as follows:

$$
g=\left(S \frac{\partial M}{\partial S}+Q \frac{\partial M}{\partial Q}\right)\left(-\frac{\partial^{2} M}{\partial S^{2}} d S^{2}+\frac{\partial^{2} M}{\partial Q^{2}} d Q^{2}\right) .
$$

Utilizing (6) and (9), we can easily calculate the relevant quantities in (24) as

$$
\begin{gathered}
\frac{\partial M}{\partial S}=\frac{r_{+}^{2}+2 \widetilde{\alpha}-Q^{2}}{4 \pi r_{+}\left(r_{+}^{2}-4 \widetilde{\alpha}\right)}, \\
\frac{\partial M}{\partial Q}=\frac{Q}{r_{+}}, \\
\frac{\partial^{2} M}{\partial S^{2}}=\frac{8 \widetilde{\alpha}^{2}-r_{+}^{4}-10 r_{+}^{2} \widetilde{\alpha}-Q^{2}\left(4 \widetilde{\alpha}-3 r_{+}^{2}\right)}{8 \pi^{2} r_{+}\left(r_{+}^{2}-4 \widetilde{\alpha}\right)^{3}}, \\
\frac{\partial^{2} M}{\partial Q^{2}}=\frac{1}{r_{+}} .
\end{gathered}
$$

Comparing (25) and (26) with (7) and (8), we find

$$
\frac{\partial M}{\partial S}=T, \quad \frac{\partial M}{\partial Q}=\Phi,
$$

which proves the validity of the first law of black hole thermodynamics $d M=T d S+\Phi d Q$. Substituting (25)-(28) into (24), we can calculate the component of the thermodynamic metric $g$ as

$g_{S S}$

$$
\begin{aligned}
= & \frac{1}{32 \pi^{2} r_{+}^{2}\left(r_{+}^{2}-4 \widetilde{\alpha}\right)^{4}} \\
& \times\left[r_{+}^{4}-8 \widetilde{\alpha}^{2}+10 r_{+}^{2} \widetilde{\alpha}+Q^{2}\left(4 \widetilde{\alpha}-3 r_{+}^{2}\right)\right] \\
& \times\left[r_{+}^{4}+2 r_{+}^{2} \widetilde{\alpha}+Q^{2}\left(3 r_{+}^{2}-16 \widetilde{\alpha}\right)+8 \widetilde{\alpha}\left(Q^{2}-r_{+}^{2}-2 \widetilde{\alpha}\right) \ln r_{+}\right],
\end{aligned}
$$

$g_{\mathrm{QQ}}$

$=\frac{r_{+}^{4}-16 Q^{2} \widetilde{\alpha}+2 r_{+}^{2} \widetilde{\alpha}+3 Q^{2} r_{+}^{2}+\left(8 Q^{2} \widetilde{\alpha}-8 r_{+}^{2} \widetilde{\alpha}-16 \widetilde{\alpha}^{2}\right) \ln r_{+}}{4 r_{+}^{2}\left(r_{+}^{2}-4 \widetilde{\alpha}\right)}$.

Utilizing (30), we can obtain the Legendre invariant scalar curvature as

$$
\mathfrak{R}_{\mathrm{Q}}=\frac{A\left(x_{+}, Q\right)}{B\left(x_{+}, Q\right)},
$$

where

$$
\begin{aligned}
& B\left(x_{+}, Q\right) \\
& =\left[r_{+}^{4}+10 r_{+}^{2} \widetilde{\alpha}-8 \widetilde{\alpha}^{2}+Q^{2}\left(4 \widetilde{\alpha}-3 r_{+}^{2}\right)\right]^{2} \\
& \times\left[r_{+}^{4}+Q^{2}\left(3 r_{+}^{2}-16 \widetilde{\alpha}\right)+2 r_{+}^{2} \widetilde{\alpha}+8 \widetilde{\alpha}\left(Q^{2}-r_{+}^{2}-2 \widetilde{\alpha}\right) \ln r_{+}\right]^{3} .
\end{aligned}
$$




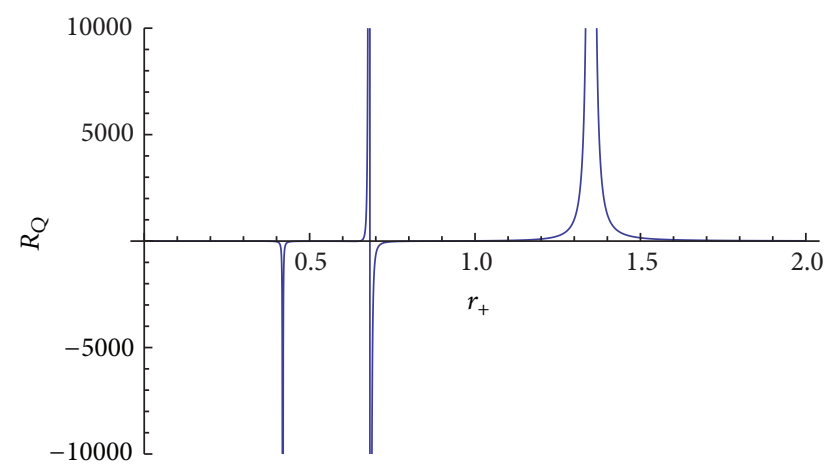

Figure 5: Thermodynamic scalar curvature $R_{Q}$ versus $r_{+}$for $Q=$ $1, \tilde{\alpha}=0.1$.

The numerator of the Legendre invariant scalar curvature is too lengthy to be displayed here. From (32), we find that the Legendre invariant scalar curvature shares the same factor $r_{+}^{4}+10 r_{+}^{2} \tilde{\alpha}-8 \widetilde{\alpha}^{2}+Q^{2}\left(4 \tilde{\alpha}-3 r_{+}^{2}\right)$ with the specific heat $C_{Q}$ in its denominator, which implies that it would diverge when $r_{+}^{4}+10 r_{+}^{2} \widetilde{\alpha}-8 \widetilde{\alpha}^{2}+Q^{2}\left(4 \widetilde{\alpha}-3 r_{+}^{2}\right)=0$. That is the exact condition that the phase transition point satisfies. To get an intuitive sense on this issue, we plot Figure 5 showing the behavior of thermodynamic scalar curvature $\mathfrak{R}_{\mathrm{Q}}$. From Figure 5 , we find that thermodynamic scalar curvature $\mathfrak{R}_{Q}$ diverges at three locations. Comparing Figure 5 with Figure 1(b), we find that the second diverging point which corresponds to negative Hawking temperature does not have physical meaning. Furthermore, the first and the third diverging points coincide exactly with the phase transition point, which can be witnessed by comparing Figure 5 with Figure 1(a). So we can safely draw the conclusion that the Legendre invariant metric constructed in geometrothermodynamics correctly produces the behavior of the thermodynamic interaction and phase transition structure of black holes with conformal anomaly.

\section{Critical Exponents and Scaling Laws}

In order to have a better understanding of the phase transition of black holes with conformal anomaly, we would like to investigate their critical behavior near the critical point by considering a set of critical exponents in this section.

Before embarking on calculating critical exponents, we would like to reexpress physical quantities near the critical point as

$$
\begin{gathered}
r_{+}=r_{c}(1+\Delta), \\
T\left(r_{+}\right)=T_{c}(1+\varepsilon), \\
Q\left(r_{+}\right)=Q_{c}(1+\eta),
\end{gathered}
$$

where $|\Delta| \ll 1,|\varepsilon| \ll 1,|\eta| \ll 1$. Note that the subindex " $c$ " in this section denotes the value of the physical quantity (or the expression) at the critical point. For example, $T_{c}$ corresponds to the temperature at the critical point.
Critical exponent $\alpha$ is defined through

$$
C_{Q} \sim\left|T-T_{c}\right|^{-\alpha}
$$

To obtain $T-T_{c}$, we would like to carry out Taylor expansion as below:

$$
\begin{aligned}
T\left(r_{+}\right)= & T_{c}+\left[\left(\frac{\partial T}{\partial r_{+}}\right)_{\mathrm{Q}=\mathrm{Q}_{c}}\right]_{r_{+}=r_{c}}\left(r_{+}-r_{c}\right) \\
& +\frac{1}{2}\left[\left(\frac{\partial^{2} T}{\partial r_{+}^{2}}\right)_{\mathrm{Q}=\mathrm{Q}_{c}}\right]_{r_{+}=r_{c}}\left(r_{+}-r_{c}\right)^{2}
\end{aligned}
$$

+ higher order terms,

from which we obtain

$$
\Delta=\frac{1}{r_{c}} \sqrt{\frac{2 \varepsilon T_{c}}{D}},
$$

where

$$
\begin{aligned}
D & =\left[\left(\frac{\partial^{2} T}{\partial r_{+}^{2}}\right)_{\mathrm{Q}=\mathrm{Q}_{c}}\right]_{r_{+}=r_{c}} \\
& =\frac{r_{c}^{6}+24 r_{c}^{4} \widetilde{\alpha}-24 r_{c}^{2} \widetilde{\alpha}^{2}+32 \widetilde{\alpha}^{3}-2 Q_{c}^{2}\left(3 r_{c}^{4}-6 r_{c}^{2} \widetilde{\alpha}+8 \widetilde{\alpha}^{2}\right)}{2 \pi\left(r_{c}^{3}-4 r_{c} \widetilde{\alpha}\right)^{3}} .
\end{aligned}
$$

In the above derivation, we have considered the fact that $C_{Q}$ diverges at the critical point, making the second term at the right hand side of (37) vanish. Substituting (33) into (10) and keeping only the linear terms in its denominator, we obtain

$$
C_{Q} \simeq \frac{2 \pi\left(r_{c}^{2}-4 \widetilde{\alpha}\right)^{2}\left(Q_{c}^{2}-2 \widetilde{\alpha}-r_{c}^{2}\right)}{\Delta\left(4 r_{c}^{4}+20 r_{c}^{2} \widetilde{\alpha}-6 Q_{c}^{2} r_{c}^{2}\right)},
$$

which can be transformed via (38) into

$$
C_{Q} \simeq \frac{\pi \sqrt{2 D}\left(r_{c}^{2}-4 \tilde{\alpha}\right)^{2}\left(Q_{c}^{2}-2 \tilde{\alpha}-r_{c}^{2}\right)}{\left(4 r_{c}^{3}+20 r_{c} \tilde{\alpha}-6 Q_{c}^{2} r_{c}\right)\left(T-T_{c}\right)^{1 / 2}}
$$

Comparing (41) with (36), we can obtain $\alpha=1 / 2$.

Critical exponent $\beta$ is defined through the following relation when $Q$ is fixed:

$$
\Phi\left(r_{+}\right)-\Phi\left(r_{c}\right) \sim\left|T-T_{c}\right|^{\beta}
$$

The above definition motivates us to carry out the Taylor expansion as

$$
\Phi\left(r_{+}\right)=\Phi_{c}+\left[\left(\frac{\partial \Phi}{\partial r_{+}}\right)_{\mathrm{Q}=Q_{c}}\right]_{r_{+}=r_{c}}\left(r_{+}-r_{c}\right)
$$

+ higher order terms. 
Utilizing (8) and (43) and neglecting higher order terms of (42), we get

$$
\begin{aligned}
\Phi\left(r_{+}\right)-\Phi_{c} & =\left[\left(\frac{\partial \Phi}{\partial r_{+}}\right)_{\mathrm{Q}=\mathrm{Q}_{c}}\right]_{r_{+}=r_{c}} \sqrt{\frac{2}{D}}\left(T-T_{c}\right)^{1 / 2} \\
& =-\frac{Q_{c}}{r_{c}^{2}} \sqrt{\frac{2}{D}}\left(T-T_{c}\right)^{1 / 2} .
\end{aligned}
$$

Comparing (42) with (44), we can obtain $\beta=1 / 2$. relation:

Critical exponent $\gamma$ is defined through the following

$$
\kappa_{T}^{-1} \sim\left|T-T_{c}\right|^{-\gamma} .
$$

Substituting (33) and (38) into (18) and keeping only the linear term of $\Delta$, we obtain

$$
\kappa_{T}^{-1}=\frac{\sqrt{D}\left(Q_{c} r_{c}^{4}-Q_{c}^{3} r_{c}^{2}-4 Q_{c}^{3} \widetilde{\alpha}+10 Q_{c} r_{c}^{2} \widetilde{\alpha}-8 Q_{c} \widetilde{\alpha}^{2}\right)}{\sqrt{2}\left[5 r_{c}^{4}-8 \widetilde{\alpha}^{2}+30 r_{c}^{2} \widetilde{\alpha}+Q_{c}^{2}\left(4 \widetilde{\alpha}-9 r_{c}^{2}\right)\right]\left(T-T_{c}\right)^{1 / 2}} .
$$

From (45) and (46), we find that $\gamma=1 / 2$.

Critical exponent $\delta$ is defined for the fixed temperature $T_{c}$ through

$$
\Phi\left(r_{+}\right)-\Phi\left(r_{c}\right) \sim\left|Q-Q_{c}\right|^{1 / \delta} .
$$

To obtain $Q-Q_{c}$, we would like to carry out Taylor expansion as

$$
\begin{aligned}
Q\left(r_{+}\right)= & Q_{c}+\left[\left(\frac{\partial Q}{\partial r_{+}}\right)_{T}\right]_{r_{+}=r_{c}}\left(r_{+}-r_{c}\right) \\
& +\frac{1}{2}\left[\left(\frac{\partial^{2} Q}{\partial r_{+}^{2}}\right)_{T}\right]_{r_{+}=r_{c}}\left(r_{+}-r_{c}\right)^{2}
\end{aligned}
$$

+ higher order terms.

Utilizing the thermodynamic identity again, we get

$$
\left[\left(\frac{\partial Q}{\partial r_{+}}\right)_{T}\right]_{r_{+}=r_{c}}=-\left[\left(\frac{\partial T}{\partial r_{+}}\right)_{Q}\right]_{r_{+}=r_{c}}\left[\left(\frac{\partial Q}{\partial T}\right)_{r_{+}}\right]_{r_{+}=r_{c}}=0 .
$$

In the above derivation, we have taken into account the fact that $C_{Q}$ diverges at the critical point, making the first term at the right hand side of (37) vanish. Substituting (33) and (35) into (48) and neglecting the high order terms, we obtain

$$
\Delta=\sqrt{\frac{2 Q_{c} \eta}{E r_{c}^{2}}}
$$

where

$$
\begin{aligned}
E & =\left[\left(\frac{\partial^{2} Q}{\partial r_{+}^{2}}\right)_{T}\right]_{r_{+}=r_{c}} \\
& =\frac{22 r_{c}^{4} \widetilde{\alpha}+32 \widetilde{\alpha}^{3}+16 \widetilde{\alpha}^{2}\left(r_{c}^{2}-Q_{c}^{2}\right)-r_{c}^{4}\left(3 Q_{c}^{2}+r_{c}^{2}\right)}{2 Q_{c}\left(r_{c}^{3}-4 r_{c} \widetilde{\alpha}\right)^{2}} .
\end{aligned}
$$

Performing Taylor expansion of the function $\Phi$ near the critical point, we get

$$
\begin{aligned}
\Phi\left(r_{+}\right)= & \Phi_{c}+\left[\left(\frac{\partial \Phi}{\partial r_{+}}\right)_{T}\right]_{r_{+}=r_{c}}\left(r_{+}-r_{c}\right) \\
& + \text { higher order terms, }
\end{aligned}
$$

where the coefficient of the second term on the right hand side can be derived as follows:

$$
\begin{aligned}
{\left[\left(\frac{\partial \Phi}{\partial r_{+}}\right)_{T}\right]_{r_{+}=r_{c}}=} & {\left[\left(\frac{\partial Q}{\partial r_{+}}\right)_{T}\right]_{r_{+}=r_{c}}\left[\left(\frac{\partial \Phi}{\partial Q}\right)_{r_{+}}\right]_{r_{+}=r_{c}} } \\
& +\left[\left(\frac{\partial \Phi}{\partial r_{+}}\right)_{Q}\right]_{r_{+}=r_{c}}=-\frac{Q_{c}}{r_{c}^{2}} .
\end{aligned}
$$

Utilizing (50), (52), and (53), we get

$$
\Phi\left(r_{+}\right)-\Phi_{c} \simeq-\frac{Q_{c}}{r_{c}^{2}} \sqrt{\frac{2\left(Q-Q_{c}\right)}{E}},
$$

from which we can draw the conclusion that $\delta=2$.

Critical exponent $\varphi$ is defined through

$$
C_{Q} \sim\left|Q-Q_{c}\right|^{-\varphi} \text {. }
$$

Substituting (50) into (40), we obtain

$$
C_{Q} \simeq \frac{\pi r_{c} \sqrt{2 E}\left(r_{c}^{2}-4 \widetilde{\alpha}\right)^{2}\left(Q_{c}^{2}-2 \widetilde{\alpha}-r_{c}^{2}\right)}{\sqrt{Q-Q_{c}}\left(4 r_{c}^{4}+20 r_{c}^{2} \widetilde{\alpha}-6 Q_{c}^{2} r_{c}^{2}\right)} .
$$

Comparing (56) and (55), we find that $\varphi=1 / 2$.

Critical exponent $\psi$ is defined through

$$
S\left(r_{+}\right)-S_{c} \sim\left|Q-Q_{c}\right|^{\psi} .
$$

Performing Taylor expansion of the function $S$ near the critical point, we obtain

$$
S\left(r_{+}\right)=S_{c}+\left[\left(\frac{\partial S}{\partial r_{+}}\right)_{\mathrm{Q}}\right]_{r_{+}=r_{c}}\left(r_{+}-r_{c}\right)+\text { higher order terms. }
$$

Utilizing (9), (33), (50), and (58), we get

$$
S\left(r_{+}\right)-S_{c} \simeq\left(2 \pi r_{c}-\frac{8 \pi \tilde{\alpha}}{r_{c}}\right) \sqrt{\frac{2\left(Q-Q_{c}\right)}{E}},
$$

from which we obtain $\psi=1 / 2$.

Till now, we have finished the calculations of six critical exponents. They are also equal to $1 / 2$ except $\delta=2$. Our results are in accordance with those in classical thermodynamics. And it can be easily proved that critical exponents we obtain in our paper satisfy the following thermodynamic scaling laws:

$$
\begin{gathered}
\alpha+2 \beta+\gamma=2, \quad \alpha+\beta(\delta+1)=2, \\
(2-\alpha)(\delta \psi-1)+1=(1-\alpha) \delta, \\
\gamma(\delta+1)=(2-\alpha)(\delta-1), \quad \gamma=\beta(\delta-1), \\
\varphi+2 \psi-\delta^{-1}=1 .
\end{gathered}
$$




\section{Conclusions}

The phase transition of black holes with conformal anomaly has been investigated in canonical ensemble. Firstly, we calculate the relevant thermodynamic quantities and discuss the behavior of the specific heat at constant charge. We find that there have been striking differences between black holes with conformal anomaly and those without conformal anomaly. In the case $Q=1, \tilde{\alpha}=0.1$, there are two phase transition points while there is only one in the case $\widetilde{\alpha}=0$. The temperature in the case $\tilde{\alpha}=0$ increases monotonically while there exists local minimum temperature in the case $Q=1, \widetilde{\alpha}=0.1$. This local minimum temperature corresponds to the phase transition point. We also find that the phase transitions of black holes with conformal anomaly take place not only from an unstable large black hole to a locally stable medium black hole but also from an unstable medium black hole to a locally stable small black hole. We also study the behavior of the inverse of the isothermal compressibility $\kappa_{T}^{-1}$ and find that $\kappa_{T}^{-1}$ also diverges at the critical point.

Secondly, we probe the dependence of phase transitions on the choice of parameters. The results show that black holes with conformal anomaly have much richer phase structure than that without conformal anomaly. When $\widetilde{\alpha}=0$, the location of the phase transition $r_{c}$ is proportional to the charge $Q$. By contrast, the case of black holes with conformal anomaly is more complicated. For $\widetilde{\alpha}=0.1$, the curve can be divided into three regions. Through numerical calculation, we find that black holes have only one phase transition point when $Q \subset(0,0.4472)$. When $0.4472<Q<0.7746$, there would be no phase transition at all. When $Q>0.7746$, there exist two phase transition points. And the distance between these two phase transition points becomes larger with the increasing of $Q$. In the case that the charge $Q$ has been fixed at one, we notice that there would be two phase transition points when $0<\widetilde{\alpha}<1 / 6$. And the distance between these two phase transition points becomes narrower with the increasing of $\widetilde{\alpha}$. When $\tilde{\alpha} \subset(1 / 6,1 / 2)$, there would be no phase transition. When $\tilde{\alpha}>1 / 2$, there would be only one phase transition point.

Thirdly, we build up geometrothermodynamics in canonical ensembles. We choose $M$ to be the thermodynamic potential and build up both thermodynamic phase space and the space of thermodynamic equilibrium states. We also calculate the Legendre invariant thermodynamic scalar curvature and depict its behavior graphically. It is shown that Legendre invariant thermodynamic scalar curvature diverges exactly where the specific heat diverges. Based on this, we can safely conclude that the Legendre invariant metrics constructed in geometrothermodynamics can correctly produce the behavior of the thermodynamic interaction and phase transition structure even when conformal anomaly is taken into account.

Furthermore, we calculate the relevant critical exponents. They are also equal to $1 / 2$ except $\delta=2$. Our results are in accordance with those of other black holes. And it has been proved that critical exponents we obtain in our paper satisfy the thermodynamic scaling laws. We conclude that the critical exponents and the scaling laws do not change even when we consider conformal anomaly. This may be attributed to the mean field theory.

\section{Conflict of Interests}

The authors declare that there is no conflict of interests regarding the publication of this paper.

\section{Acknowledgments}

This research is supported by the National Natural Science Foundation of China (Grants nos. 11235003, 11175019, and 11178007). It is also supported by "Thousand Hundred Ten" Project of Guangdong Province and Natural Science Foundation of Zhanjiang Normal University under Grant no. QL1104.

\section{References}

[1] J. D. Bekenstein, "Black holes and entropy," Physical Review D, vol. 7, no. 8, pp. 2333-2346, 1973.

[2] S. W. Hawking, "Particle creation by black holes," Communications in Mathematical Physics, vol. 43, p. 199, 1975.

[3] S. W. Hawking and D. N. Page, "Thermodynamics of black holes in anti-de Sitter space," Communications in Mathematical Physics, vol. 87, no. 4, pp. 577-588, 1983.

[4] J. Maldacena, "The large N Limit of superconformal field theories and supergravity," Advances in Theoretical and Mathematical Physics, vol. 2, no. 2, pp. 231-252, 1998.

[5] E. Witten, "Anti-de Sitter space, thermal phase transition, and confinement in gauge theories," Advances in Theoretical and Mathematical Physics, vol. 2, no. 3, pp. 505-532, 1998.

[6] A. Sahay, T. Sarkar, and G. Sengupta, "On the phase structure and thermodynamic geometry of R-charged black holes," Journal of High Energy Physics, vol. 2010, article 125, 2010.

[7] R. Banerjee, S. K. Modak, and S. Samanta, "Glassy phase transition and stability in black holes," European Physical Journal C, vol. 70, no. 1, pp. 317-328, 2010.

[8] R. Banerjee, S. K. Modak, and S. Samanta, "Second order phase transition and thermodynamic geometry in Kerr-AdS black holes," Physical Review D, vol. 84, no. 6, Article ID 064024, 2011.

[9] Q.-J. Cao, Y.-X. Chen, and K.-N. Shao, "Black hole phase transitions in Hořava-Lifshitz gravity," Physical Review D, vol. 83, no. 6, Article ID 064015, 2011.

[10] R. Banerjee and D. Roychowdhury, "Critical phenomena in Born-Infeld AdS black holes," Physical Review D, vol. 85, no. 4, Article ID 044040, 2012.

[11] R. Banerjee, S. Ghosh, and D. Roychowdhury, "New type of phase transition in Reissner Nordström-AdS black hole and its thermodynamic geometry," Physics Letters B, vol. 696, no. 1-2, pp. 156-162, 2011.

[12] R. Banerjee and D. Roychowdhury, "Thermodynamics of phase transition in higher dimensional AdS black holes," Journal of High Energy Physics, vol. 2011, article 4, 2011.

[13] R. Banerjee, S. K. Modak, and D. Roychowdhury, "A unified picture of phase transition: from liquid-vapour systems to AdS black holes," Journal of High Energy Physics, vol. 2012, article 125, 2012. 
[14] S. W. Wei and Y. X. Liu, "Thermodynamic geometry of black hole in the deformed Horava-Lifshitz gravity," Europhysics Letters, vol. 99, no. 2, Article ID 20004, 2012.

[15] B. R. Majhi and D. Roychowdhury, "Phase transition and scaling behavior of topological charged black holes in Horava- Lifshitz gravity," Class. Quantum Grav, vol. 29, no. 24, Article ID 245012, 2012.

[16] W. Kim and Y. Kim, "Phase transition of quantum corrected Schwarzschild black hole," Physics Letters B, vol. 718, no. 2, pp. 687-691, 2012.

[17] Y.-D. Tsai, X. N. Wu, and Y. Yang, "Phase structure of the KerrAdS black hole," Physical Review D, vol. 85, no. 4, Article ID 044005, 2012.

[18] F. Capela and G. Nardini, "Hairy black holes in massive gravity: thermodynamics and phase structure," Physical Review D, vol. 86, no. 2, Article ID 024030, 12 pages, 2012.

[19] D. Kubiznak and R. B. Mann, " $P-V$ criticality of charged AdS black holes," Journal of High Energy Physics, vol. 2012, article 33, 2012.

[20] C. Niu, Y. Tian, and X.-N. Wu, "Critical phenomena and thermodynamic geometry of RN-AdS black holes," Physical Review D, vol. 85, no. 2, Article ID 024017, 2012.

[21] A. Lala and D. Roychowdhury, "Ehrenfests scheme and thermodynamic geometry in Born-Infeld AdS black holes," Physical Review D, vol. 86, no. 8, Article ID 084027, 8 pages, 2012.

[22] A. Lala, "Critical phenomena in higher curvature charged AdS black holes," Advances in High Energy Physics, vol. 2013, Article ID 918490, 18 pages, 2013.

[23] S. W. Wei and Y. X. Liu, "Critical phenomena and thermodynamic geometry of charged Gauss-Bonnet AdS black holes," Physical Review D, vol. 87, no. 4, Article ID 044014, 14 pages, 2013.

[24] M. Eune, W. Kim, and S. H. Yi, "Hawking-Page phase transition in BTZ black hole revisited," Journal of High Energy Physics, vol. 2013, article 20, 2013.

[25] M. B. J. Poshteh, B. Mirza, and Z. Sherkatghanad, "Phase transition, critical behavior, and critical exponents of MyersPerry black holes," Physical Review D, vol. 88, no. 2, Article ID 024005, 8 pages, 2013.

[26] J. X. Mo and W. B. Liu, "Ehrenfest scheme for $P-V$ criticality in the extended phase space of black holes," Physics Letters B, vol. 727, pp. 336-339, 2013.

[27] F. Weinhold, "Metric geometry of equilibrium thermodynamics," The Journal of Chemical Physics, vol. 63, no. 6, pp. 24792483, 1975.

[28] G. Ruppeiner, "Thermodynamics: a Riemannian geometric model," Physical Review A, vol. 20, no. 4, pp. 1608-1613, 1979.

[29] H. Janyszek and R. Mrugała, "Geometrical structure of the state space in classical statistical and phenomenological thermodynamics," Reports on Mathematical Physics, vol. 27, no. 2, pp. 145159, 1989.

[30] G. Ruppeiner, “Thermodynamic curvature and black holes," http://arxiv.org/abs/1309.0901.

[31] H. Quevedo, "Geometrothermodynamics," Journal of Mathematical Physics, vol. 48, no. 1, Article ID 013506, 2007.

[32] H. Quevedo, "Geometrothermodynamics of black holes," General Relativity and Gravitation, vol. 40, no. 5, pp. 971-984, 2008.

[33] H. Quevedo and A. Snchez, "Geometrothermodynamics of asymptotically Anti-de Sitter black holes," Journal of High Energy Physics, vol. 2008, 34, 2008.
[34] J. L. Álvarez, H. Quevedo, and A. Sánchez, "Unified geometric description of black hole thermodynamics," Physical Review D, vol. 77, no. 8, Article ID 084004, 2008.

[35] H. Quevedo and A. Sánchez, "Geometrothermodynamics of black holes in two dimensions," Physical Review D, vol. 79, no. 8, Article ID 087504, 2009.

[36] H. Quevedo and A. Sánchez, "Geometric description of BTZ black hole thermodynamics," Physical Review D, vol. 79, no. 2, Article ID 024012, 2009.

[37] M. Akbar, H. Quevedo, K. Saifullah, A. Sánchez, and S. Taj, "Thermodynamic geometry of charged rotating BTZ black holes," Physical Review D, vol. 83, no. 8, Article ID 084031, 2011.

[38] H. Quevedo, A. Sánchez, S. Taj, and A. Vázquez, "Phase transitions in geometrothermodynamics," General Relativity and Gravitation, vol. 43, no. 4, pp. 1153-1165, 2011.

[39] A. Aviles, A. B. Almodovar, L. Campuzano, and H. Quevedo, "Extending the generalized Chaplygin gas model by using geometrothermodynamics," Physical Review D, vol. 86, no. 6, Article ID 063508, 10 pages, 2012.

[40] Y. W. Han and G. Chen, "Thermodynamics, geometrothermodynamics and critical behavior of $(2+1)$-dimensional black hole," Physics Letters B, vol. 714, pp. 127-130, 2012.

[41] J. X. Mo, X. X. Zeng, G. Q. Li, X. Jiang, and W. B. Liu, "A unified phase transition picture of the charged topological black hole in Hořava-Lifshitz gravity," Journal of High Energy Physics, vol. 2013, article 56, 2013.

[42] P. C. W. Davies, "Thermodynamic phase transitions of KerrNewman black holes in de Sitter space," Classical and Quantum Gravity, vol. 6, no. 12, article 018, pp. 1909-1914, 1989.

[43] C. O. Lousto, "The fourth law of black-hole thermodynamics," Nuclear Physics B, vol. 410, no. 1, pp. 155-172, 1993.

[44] Y. K. Lau, "On the second order phase transition of a ReissnerNordstrom black hole," Physics Letters A, vol. 186, no. 1-2, pp. 41-46, 1994.

[45] C. O. Lousto, "Emergence of an effective two-dimensional quantum description from the study of critical phenomena in black holes," Physical Review D, vol. 51, no. 4, pp. 1733-1740, 1995.

[46] R.-G. Cai and Y. S. Myung, "Critical behavior for the dilaton black holes," Nuclear Physics B, vol. 495, no. 1-2, pp. 339-362, 1997.

[47] C. O. Lousto, "Some thermodynamic aspects of black holes and singularities," International Journal of Modern Physics D, vol. 6, no. 5, pp. 575-590, 1997.

[48] G. Arcioni and E. Lozano-Tellechea, "Stability and critical phenomena of black holes and black rings," Physical Review D, vol. 72, no. 10, Article ID 104021, 2005.

[49] J. P. Muniain and D. Píriz, "Critical behavior of dimensionally continued black holes," Physical Review D, vol. 53, no. 2, pp. 816823, 1996.

[50] R.-G. Cai, Z.-J. Lu, and Y.-Z. Zhang, "Critical behavior in (2+1)dimensional black holes," Physical Review D, vol. 55, no. 2, pp. 853-860, 1997.

[51] X. N. Wu, "Multicritical phenomena of Reissner-Nordström antiCde Sitter black holes," Physical Review D, vol. 62, Article ID 124023, 2000.

[52] S. Carlip and S. Vaidya, "Phase transitions and critical behaviour for charged black holes," Classical and Quantum Gravity, vol. 20, no. 16, pp. 3827-3837, 2003.

[53] K. Maeda, M. Natsuume, and T. Okamura, "Dynamic critical phenomena in the AdS/CFT duality," Physical Review D, vol. 78, no. 10, Article ID 106007, 2008. 
[54] S. Jain, S. Mukherji, and S. Mukhopadhyay, "Notes on R-charged black holes near criticality and gauge theory," Journal of High Energy Physics, vol. 2009, article 51, 2009.

[55] Y. Liu, Q. Pan, B. Wang, and R.-G. Cai, "Dynamical perturbations and critical phenomena in Gauss-Bonnet AdS black holes," Physics Letters B, vol. 693, no. 3, pp. 343-350, 2010.

[56] R.-G. Cai, L.-M. Caob, and N. Ohtab, "Black holes in gravity with conformal anomaly and logarithmic term in black hole entropy," Journal of High Energy Physics, vol. 2010, article 82, 2010.

[57] E. J. Son and W. Kim, "Two critical phenomena in the exactly soluble quantized Schwarzschild black hole," Journal of High Energy Physics, vol. 2013, article 60, 2013.

[58] R. Li, "Logarithmic entropy of black hole in gravity with conformal anomaly from quantum tunneling approach," Europhysics Letters, vol. 96, no. 6, Article ID 60014, 2011.

[59] J. Man and H. Cheng, "Thedescription of phase transition in a black hole with conformal anomaly in the Ehrenfest's scheme," http://arxiv.org/abs/1304.5685. 

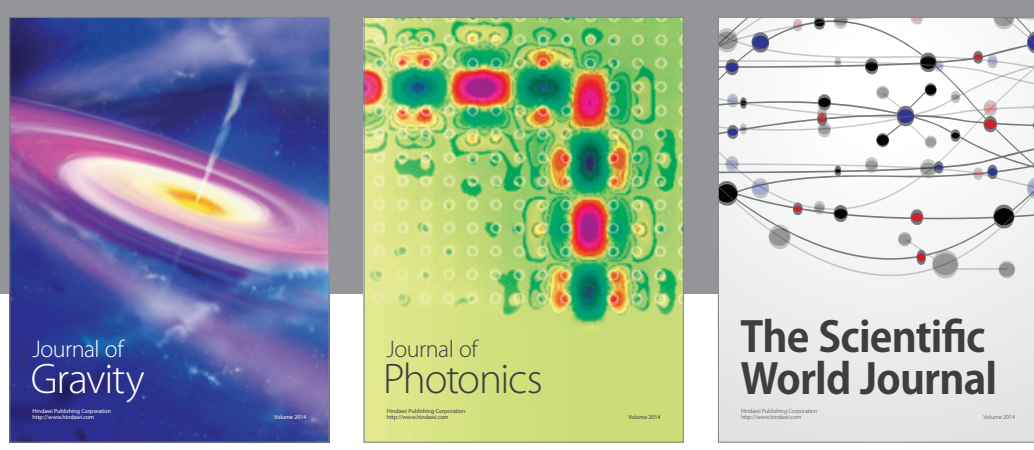

The Scientific World Journal
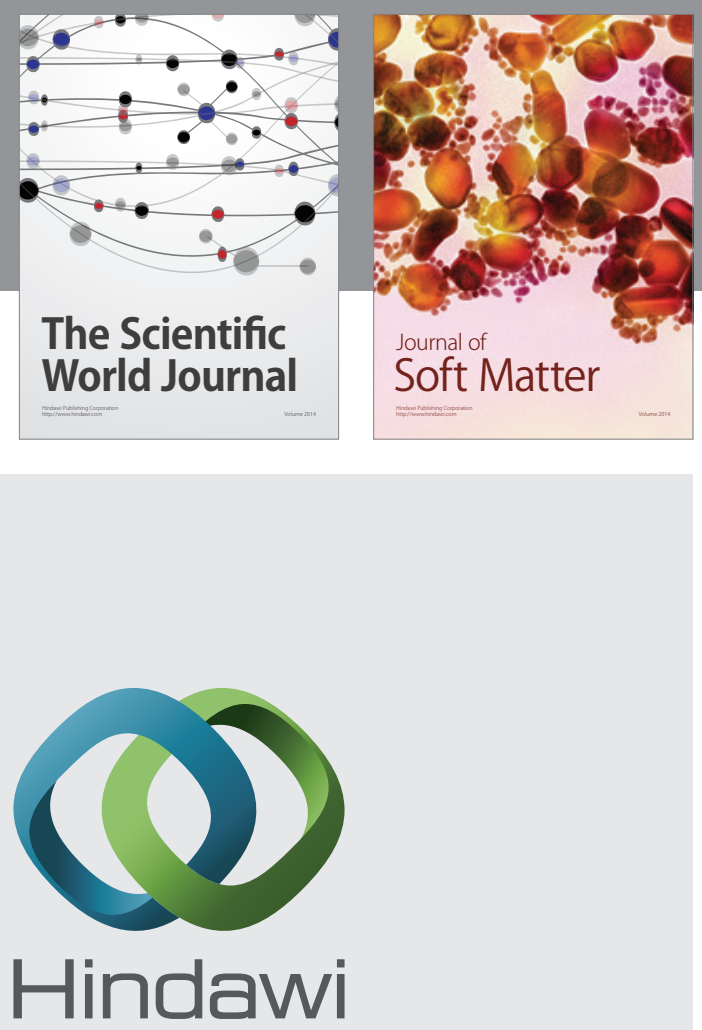

Submit your manuscripts at

http://www.hindawi.com

nternational Journal of

Statistical Mechanics
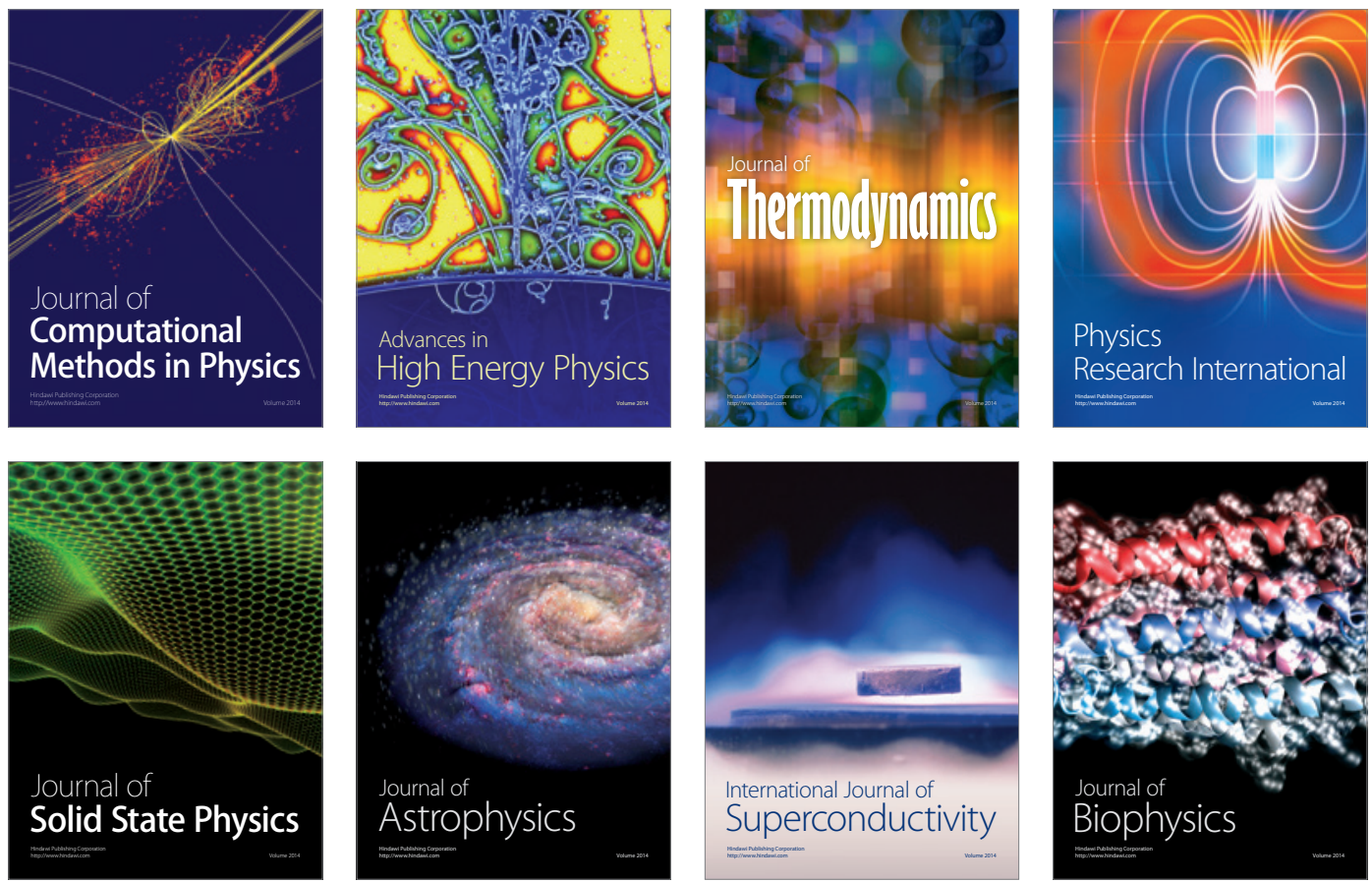
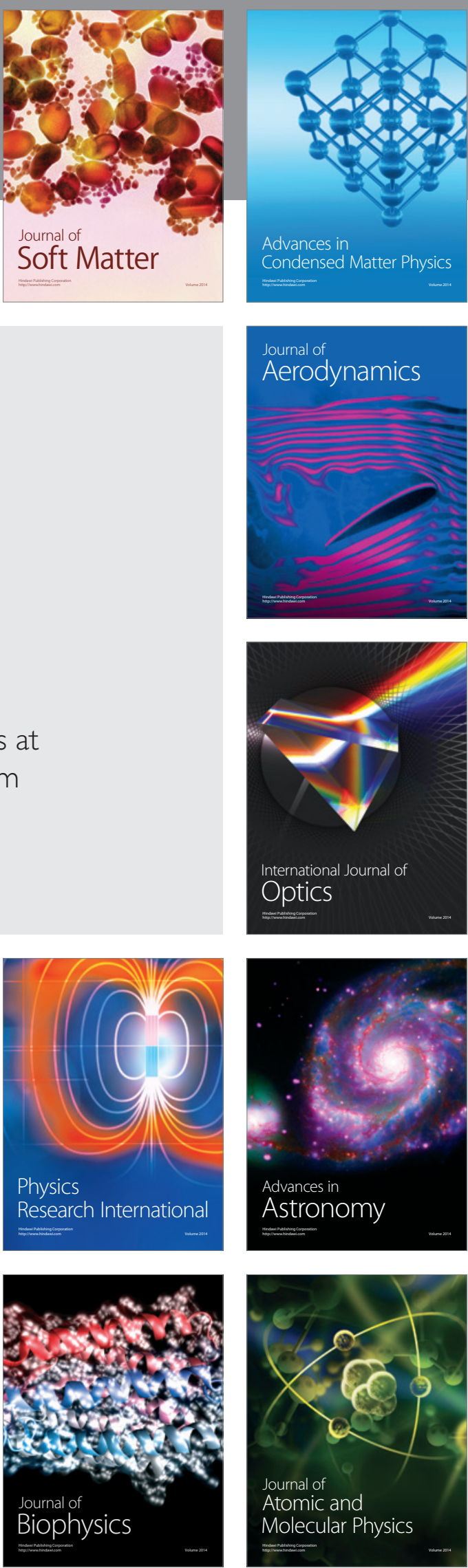